\title{
Sialolithiasis of minor salivary gland: a challenging diagnostic dilemma
}

\author{
Apostolos Matiakis ${ }^{1}$, Fotios Tzermpos ${ }^{2}$ \\ ${ }^{\prime}$ Department of Oral Medicine and Pathology, School of Dentistry, Aristotle University of Thessaloniki, Thessaloniki, \\ ${ }^{2}$ Department of Oral and Maxillofacial Surgery, School of Dentistry, University of Athens, Athens, Greece
}

\begin{abstract}
J Korean Assoc Oral Maxillofac Surg 2021;47:145-148)
Minor salivary gland sialolithiasis (MSGS) is a not uncommon oral mucosal disease. Its clinical appearance may mimic a mucocyst or other benign submucosal overgrowth. Stasis of saliva, which accompanies MSGS, usually results in minor salivary gland inflammation, with a chronic sialadenitis appearance. MSGS typically is a painless lesion but can become painful when the salivary gland parenchyma or excretory duct becomes infected, with or without pus. However, misdiagnosis of this condition is rather common, as the clinical appearance is asymptomatic. The most common location is the upper lip, and MSGS affects males and females, with a slight predilection for males. The sialolith causing MSGS may be obvious during surgical excision, as in the case reported. In other cases, sialolith may be absent or fragmented. Differential diagnosis includes mucocele, swelling due to local irritation like fibroma and diapneusia, chronic abscess of the oral mucosa, and neoplasms either benign (lymphangioma, pleiomorphic adenoma) or malignant. Histopathological examination is needed to establish clinical diagnosis.
\end{abstract}

Key words: Sialolithiasis, Minor salivary gland

\section{Introduction}

Salivary glands sialolithiasis is a common oral disease that occurs more commonly than minor salivary gland sialolithiasis (MSGS). However, MSGS has been found to have a higher incidence than originally believed ${ }^{1-3}$.

Many MSGS cases, being asymptomatic or small in size, may be missed during an intraoral examination ${ }^{1}$. In addition, some cases may be misdiagnosed as a common mucocele or a fibroma due to irritation or as a diapneusia ${ }^{3}$. Consequently, the patient may not undergo a histopathological examination, which is needed to establish the MSGS diagnosis.

In this paper, a case of MSGS located in the upper lip mucosa, primarily diagnosed as a mucocele, is reported.

\footnotetext{
Apostolos Matiakis

Department of Oral Medicine and Pathology, School of Dentistry, Aristotle University of Thessaloniki, 93, Tsimiski street, Thessaloniki 54622, Greece TEL: +30-2310-236207

E-mail:amatiakis@dent.auth.gr

ORCID: https://orcid.org/0000-0002-9314-3531

(c) This is an open-access article distributed under the terms of the Creative Commons Attribution Non-Commercial License (http://creativecommons.org/ licenses/by-nc/4.0/), which permits unrestricted non-commercial use, distribution, and reproduction in any medium, provided the original work is properly cited. Copyright (C) 2021 The Korean Association of Oral and Maxillofacial Surgeons. All rights reserved.
}

\section{Case Report}

A 65-year-old male visited our private clinic for evaluation of a submucosal nodule on the left side of the upper lip.

The patient had undergone surgical excision of a similar mass in the same location two months prior and reported recurrence after a few weeks.

Intraoral clinical examination revealed a movable asymptomatic submucosal nodule, about $2 \mathrm{~cm}$ in diameter, on the left side of the upper lip, fluctuant in palpation. A whiteish color was visible through the mucosa, under pressure.(Fig. 1) The patient was edentulous, having undergone no dental procedure in the recent past and no injury due to mechanical irritation of the upper labial mucosa. His medical history was unremarkable. An initial diagnosis of possible mucocele was based on clinical examination and lesion appearance.

An excision was made under local anesthesia to reveal the lesion. A spherical, whitish sialolith was avulsed.(Fig. 2) The cyst-like lesion was removed in total, along with the adjacent minor salivary glands. Closure was accomplished with black silk sutures that were removed after one week. Healing was uneventful, and there has been no recurrence over two years.

Histological examination revealed chronic sialadenitis of minor salivary glands, with severe dilatation of the ducts, 


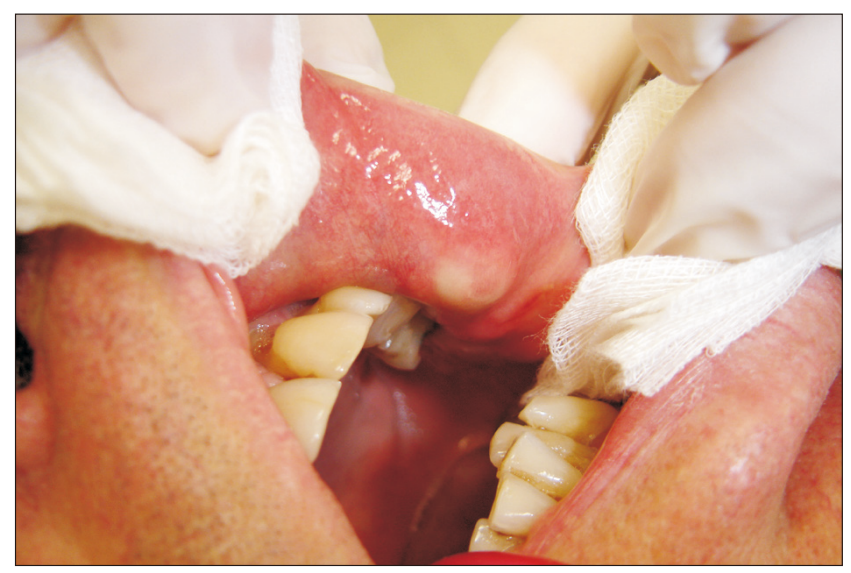

Fig. 1. A whitewish submucosal nodule, on the left side of the upper lip.

Apostolos Matiakis et al: Sialolithiasis of minor salivary gland: a challenging diagnostic dilemma. J Korean Assoc Oral Maxillofac Surg 2021

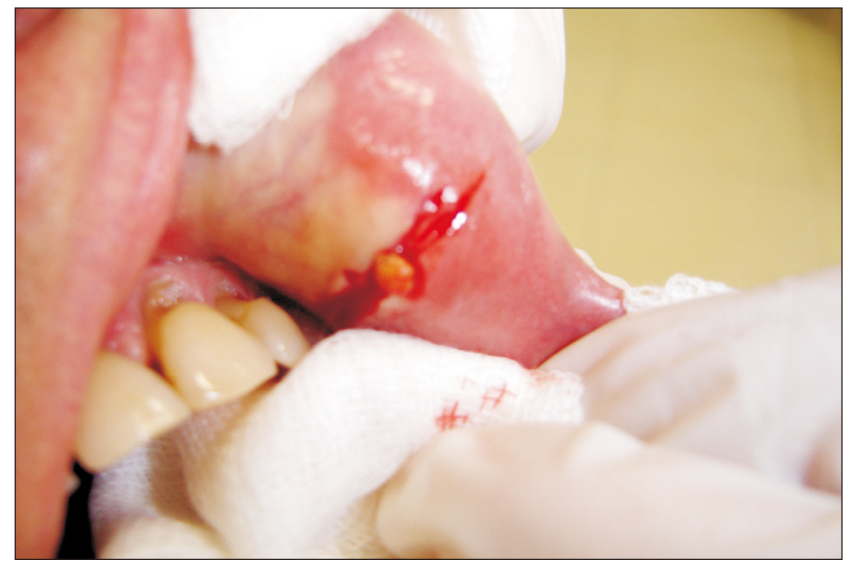

Fig. 2. A global (spherical) sialolith, whitish in color avulsed under the excision

Apostolos Matiakis et al: Sialolithiasis of minor salivary gland: a challenging diagnostic dilemma. J Korean Assoc Oral Maxillofac Surg 2021

lobular atrophy, and severe lymphocytic infiltration in the stroma with lymph follicle formation.(Fig. 3, 4)

The sialolith existence with histopathological findings established the MSGS diagnosis.

\section{Discussion}

A case of MSGS, initially diagnosed as an upper lip mucocele, is described. In the medical literature, the first MSGS case was reported by Papin (1864-1865) ${ }^{2,3}$. However, the first histopathologically established MSGS case was published much later by Lighterman in $1955^{2,3}$. Since then, some solitary MSGS cases are described as a small, hard, firm or movable asymptomatic submucosal nodule ${ }^{4}$. Holst published the first extensive report of nine cases, including the first re-

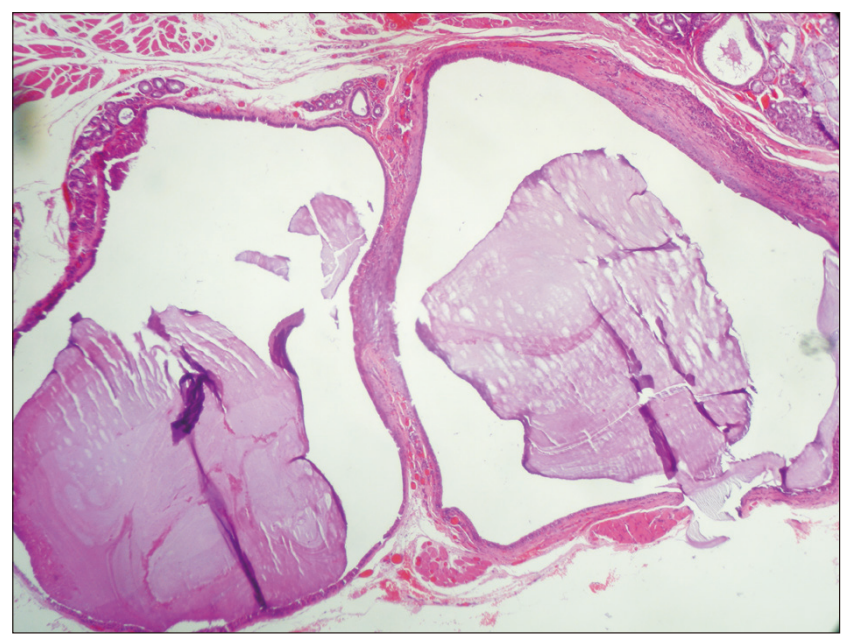

Fig. 3. Chronic sialadenitis, due to sialolithiasis, with severe dilatation of the ducts (H\&E staining, $\times 50)$.

Apostolos Matiakis et al: Sialolithiasis of minor salivary gland: a challenging diagnostic dilemma. J Korean Assoc Oral Maxillofac Surg 2021

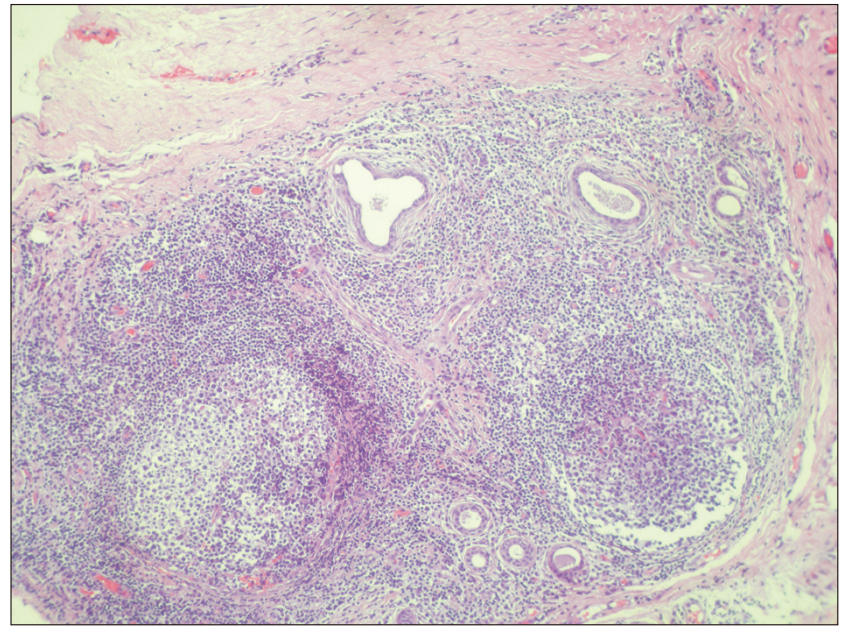

Fig. 4. Chronic sialadenitis with lobular atrophy, ductal dilatation and severe lymphocytic infiltration in stroma with lymph follicle formation (H\&E staining, $\times 100$ ).

Apostolos Matiakis et al: Sialolithiasis of minor salivary gland: a challenging diagnostic dilemma. J Korean Assoc Oral Maxillofac Surg 2021

ported tongue-located case, as well as the first with nodules in multiple locations in the same patient (two locations in the upper lip).

These data suggest that MSGS is not the uncommon entity it was believed to be ${ }^{1}$. However, misdiagnosis is rather common, as the clinical appearance of this particular disease is asymptomatic. Additionally, a sialolith may not be present during surgical excision. So, it is essential that the pathologist is informed about the specific oral mucosal lesion so that he will not overlook the existence of a sialolith or particles of it ${ }^{1}$. The existence of a micro sialolith in the acinar parenchyma 
or in the acinar duct may lead to development of a retention mycocyst.

Pullon and Miller ${ }^{2}$ performed the first MSGS review along with report of 10 new cases, for a total of 55 cases in the medical literature. The majority of these lesions was located in the buccal mucosa (24 cases), followed by the upper lip (22 cases). The male:female ratio was $2: 1$.

Anneroth and Hansen ${ }^{5}$ describe 49 new cases with similar findings. Among them, the upper lip is the most common location. The male:female ratio is similar to that reported by previous authors, with a slight predilection for males.

Similar findings to previous studies were reported in other case series $^{6,7}$.

The largest review of 239 MSGS cases by Ben Lagha et al. ${ }^{3}$ confirms that (1) the most common lesion location is the upper lip (49.2\%), followed by the buccal mucosa (37.3\%); (2) MSGS affects both males and females (58.3\% and 41.7\%), with a slight predilection for males; and (3) the mean age of affected patients is 50 years (range, 9-90 years).

Development of sialoliths is a multifactoral event. Sialoliths are composed of a central core of organic material, covered by a layered calcified cortex. That calcified cortex consists of complicated salts of calcium, phosphate, and especially hydroxyapatite ${ }^{8}$. Salivary dysfunction or disturbances of secretion may contribute to creation of sialoliths. Sialolith formation occurs through gradual calcification of an organic matrix of glycoproteins or of a foreign body. Lee and Wong ${ }^{8}$ reported an extensive study of the pathogenesis of sialolithiasis in minor salivary glands.

Concerning establishment of the diagnosis, radiographs are not essential as many of the calculi (sialolith) can be radiotransparent ${ }^{3}$. In addition, an avulsion of calculus (calculi) is possible.

Diagnostic ultrasound may help in establishing the diagnosis, as it is used to indicate major salivary gland sialoliths ${ }^{9}$. This tool may also contribute to removal of calculus or such particles with use of an ultrasound-guided needle ${ }^{9}$.

Nevertheless, ultrasound use seems to be limited in MSGS diagnosis since there is only one related paper in the literature.

Differential diagnosis includes mucocele ${ }^{6,10}$, swellings due to local irritation like fibroma and diapneusia, chronic abscess of the oral mucosa, and neoplasms either benign (lymphangioma, pleiomorphic adenoma) or malignant ${ }^{11,12}$.

Final diagnosis is clinically established in cases with sialolith avulsion noted during the surgical procedure, as in our case, but the diagnosis can be confirmed only histopathologically. Among the histological findings is the presence of a sialolith in the minor salivary gland duct or fragments of sialoliths in different stages of calcification ${ }^{13,14}$.

Stasis of saliva, which accompanies MSGS, usually results in minor salivary gland inflammation with a chronic sialadenitis appearance, as in our case. The salivary duct is dilated and surrounded by lymphocytes, plasma cells, and eosinophils $^{8,11,14}$. Squamous metaplasia and pseudostratified columnar metaplasia in duct epithelium are additional characteristic histological features ${ }^{13}$.

MSGS tends to be a painless lesion but can become painful when the salivary gland parenchyma or excretory duct becomes infected, with or without pus. In that case, antibiotic medications are recommended. An antibiotic with high diffusion in saliva, such as macrolide, is preferable ${ }^{3}$.

Surgical excision is the treatment of choice. Lithotripsy, the treatment of choice in sialolithiasis of major salivary glands ${ }^{15}$, is not applicable in MSGS. To prevent recurrence, the lesion ought to be removed surgically, along with all affected minor salivary glands.

In conclusion, MSGS is not an uncommon oral clinical entity, can mimic a mucocele, and must always be included in the differential diagnosis of swelling with or without fluctuation, especially in the upper lip. Surgical excision with affected minor salivary glands is the treatment of choice, and histological examination is needed to establish the clinical diagnosis of MSGS.

\section{ORCID}

Apostolos Matiakis, https://orcid.org/0000-0002-9314-3531

Fotios Tzermpos, https://orcid.org/0000-0002-2535-6623

\section{Authors' Contributions}

A.M. examined the patient and diagnosed the clinical entity. Also, he participated in the surgical excision of the lesion and he wrote the article. F.T. participated in the surgical excision and he also participated in the discussion of the article.

\section{Conflict of Interest}

No potential conflict of interest relevant to this article was reported. 


\section{References}

1. van der Waal I. Sialolithiasis of minor salivary glands: how rare? Report of two cases. J Oral Surg 1971;29:815-6.

2. Pullon PA, Miller AS. Sialolithiasis of accessory salivary glands: review of 55 cases. J Oral Surg 1972;30:832-4.

3. Ben Lagha N, Alantar A, Samson J, Chapireau D, Maman L. Lithiasis of minor salivary glands: current data. Oral Surg Oral Med Oral Pathol Oral Radiol Endod 2005;100:345-8. https://doi. org/10.1016/j.tripleo.2004.12.023

4. Holst E. The clinical entity of sialolithiasis of the minor salivary glands. Acta Odontol Scand 1971;29:75-84. https://doi. org/10.3109/00016357109026324

5. Anneroth G, Hansen LS. Minor salivary gland calculi. A clinical and histopathological study of 49 cases. Int J Oral Surg 1983;12:80-9. https://doi.org/10.1016/s0300-9785(83)80002-7

6. Brazao-Silva MT, Prosdocimi FC, Lemos-Junior CA, de Sousa SO. Clinicopathological aspects of 25 cases of sialolithiasis of minor salivary glands. Gen Dent 2015;63:e22-6.

7. Wang WC, Chen CY, Hsu HJ, Kuo JH, Lin LM, Chen YK. Sialolithiasis of minor salivary glands: a review of 17 cases. J Dent Sci 2016;11:152-5. https://doi.org/10.1016/j.jds.2015.10.006

8. Lee LT, Wong YK. Pathogenesis and diverse histologic findings of sialolithiasis in minor salivary glands. J Oral Maxillofac Surg 2010;68:465-70. https://doi.org/10.1016/j.joms.2009.03.041

9. Ng SY, Pinto P. Ultrasound-guided retrieval of labial minor salivary gland sialoliths. Dentomaxillofac Radiol 2000;29:319-22. https:// doi.org/10.1038/sj/dmfr/4600545

10. Okada H, Yokokawa M, Komiya M, Akimoto Y, Kaneda T, Yamamoto H. A rare case of sialolithiasis of the lower lip simulating a mucocele and review of the literature. Quintessence Int 2011;42:589-94.

11. Boyd AS. Sialolith of a minor salivary gland. J Cutan Pathol 2013;40:695-8.

12. Suh DW, Lee EJ, Lew BL, Sim WY. Minor salivary gland sialolithiasis of the upper lip. Ann Dermatol 2013;25:502-4. https://doi. org/10.5021/ad.2013.25.4.502

13. Barnett ML. Sialolithiasis of a labial gland. Oral Surg Oral Med Oral Pathol 1971;32:22-8. https://doi.org/10.1016/00304220(71)90246-5

14. Ho V, Currie WJ, Walker A. Sialolithiasis of minor salivary glands. Br J Oral Maxillofac Surg 1992;30:273-5. https://doi. org/10.1016/0266-4356(92)90275-n

15. Su CH, Lee KS, Tseng TM, Hung SH. Endoscopic holmium:YAG laser-assisted lithotripsy: a preliminary report. B-ENT 2015;11:5761

How to cite this article: Matiakis A, Tzermpos F. Sialolithiasis of minor salivary gland: a challenging diagnostic dilemma. J Korean Assoc Oral Maxillofac Surg 2021;47:145-148. https://doi. org/10.5125/jkaoms.2021.47.2.145 